\title{
Cáncer cervicouterino estadios IB2-IIB en pacientes jóvenes: resultados del tratamiento y una propuesta de manejo
}

\author{
Alfonso Torres-Lobatón ${ }^{1, *}$, Eduardo Carrillo-Nolasco ${ }^{1}$, Juan Carlos Oliva-Posada ${ }^{1}$, \\ Rosalva Barra-Martínez ${ }^{1}$, Miguel Ángel Morales-Palomares ${ }^{1}$ y Fred Morgan-Ortiz ${ }^{2}$
}

${ }^{1}$ Servicio de Oncología, Hospital General de México Dr. Eduardo Liceaga, Ciudad de México, México; ${ }^{2}$ Maestría en Ciencias, Universidad Autónoma de Sinaloa, Culiacán, Sinaloa, México

Recibido el 1 de octubre de 2016; aceptado el 25 de febrero de 2017

Disponible en Internet el 06 de noviembre de 2017

\section{PALABRAS CLAVE \\ Cáncer cervicouterino pacientes jóvenes}

\section{KEY WORDS}

Cervical Cancer

Stages IB2-IIB In young patients
Resumen Introducción: El cáncer cervicouterino $(\mathrm{CaCu})$ avanzado continúa representando en nuestro país un importante problema de salud. Un porcentaje cada vez mayor se diagnostica en mujeres jóvenes. Objetivo: Mostrar una experiencia institucional con la terapéutica convencio-z nal en pacientes menstruantes con CaCu en estadios IB2-IIB, agregando en casos indicados cirugías complementarias o de rescate y proponer para estas pacientes alguna alternativa quirúrgica de manejo. Material y métodos: Análisis de la casuística del Servicio de Oncología del Hospital General de México de pacientes de CaCu IB2-IIB con 45 años de edad o menos, que recibieron radioterapia y quimioterapia concomitante (RTQTCC) con o sin cirugía ulterior. Resultados: Se trataron 98 pacientes con edad promedio de 37.1 años. En 89 de ellas se realizó seguimiento; de las cuales $63(70.7 \%)$ evolucionaron un promedio de 30 meses sin evidencia de enfermedad: $12 / 19(63.1 \%)$ en estadio IB2 y $51 / 70(72.8 \%)$ en estadios II $(p>0.05)$. En estadio IB2, únicamente $4 / 8$ adenocarcinomas (50\%) tuvieron estos resultados, vs. $8 / 11$ (72.7\%) carcinomas epidermoides ( $p>0.05)$. En estadios II, las cifras fueron 8/11 para los adenocarcinomas, (72.7\%) y 43/59 para los epidermoides $(72.8 \%)$. Con cirugías ulteriores los resultados se mejoraron en un $10.1 \%$. Se demostró fracaso de la terapéutica establecida en 17 pacientes (19.3\%). Cuatro por progresión locorregional $(23.5 \%)$ y $13(76.4 \%)$ por diseminación a distancia. Conclusiones: Los resultados para el estadio IB2 fueron menores a los de los estadios II y la mayoría de los fracasos se relacionaron con metástasis a distancia. A fin de evitar menopausias precoces, se sugiere explorar el empleo de quimioterapia neoadyuvante más histerectomía radical en pacientes jóvenes. (creativecommons.org/licenses/by-nc-nd/4.0/).

Abstract Introduction: Advanced cervical cancer still represents a major health problem in our country. The percentage diagnosed in young women has been increasing. Objective: We present our institutional experience with conventional therapy in menstruating patients with advanced cervical cancer (stages IB2-IIB); adding in selected cases complementary or rescue surgery as indicated, and our proposal for alternative surgical management for these patients. Methods: We analyzed the cases attended in the Oncology Service, at Hospital General de

*E-mail para correspondencia: drtorreslobaton@prodigy.net.mx (A. Torres-Lobatón) 
Mexico, with cervical cancer stages IB2-IIB aged 45 years or less, who received concomitant radiotherapy and chemotherapy, with or without further surgery. Results: 98 patients were treated, with a mean age of 37.1 years. Follow up was obtained in 89 cases, and 63 cases $(70.7 \%)$ had no evidence of disease in an average follow up of 30 months: 12 of 19 cases $(63.1 \%)$ were stage IB2 and 51 of $70(72.8 \%)$ in stage II ( $7>0.05)$. In stage IB2, only 4 of 8 adenocarcinomas (50\%) had these results, compared with 8 of $11(72.7 \%)$ squamous cell carcinomas $(p>0.05)$. In stage II, the percentages were 8 of 11 for adenocarcinomas (72.7\%), and 43 of 59 for squamous cell carcinomas (72.8\%). Further surgeries in selected patients improved results in $10.1 \%$. Therapeutic failure was demonstrated in 17 patients (19.3\%): four cases by locoregional progression $(23.5 \%)$, and 13 cases $(76.4 \%)$ for distant metastasis. Conclusions: The results for the IB2 stage patients were lower than in stage II patients, and most failures were associated with distant metastases. In order to avoid early menopauses, we suggest that the use of neoadjuvant chemotherapy plus radical hysterectomy should be explored in younger patients.

\section{INTRODUCCIÓN}

El cáncer cervicouterino $(\mathrm{CaCu})$ constituye un problema de salud y representa la segunda neoplasia maligna de la mujer a nivel mundial, con más de 500,000 casos diagnosticados anualmente y más de 260,000 defunciones anuales por su causa. Es el cáncer más común del tracto genital femenino y el que ocasiona el mayor número de decesos ${ }^{1,2,3}$.

Mientras que en sus etapas tempranas los resultados terapéuticos son muy alentadores, los obtenidos en las etapas avanzadas, en las que se utiliza radioterapia como tratamiento de base, se acompañan de fracasos terapéuticas que oscilan entre un $20 \%$ a un $75 \%$ según el estadio clínico en que se diagnostique la enfermedad ${ }^{3,4}$.

Si bien el advenimiento de la quimioterapia a base de platino como agente radiosensibilizador para el tratamiento de las etapas avanzadas mejoró hasta en un $12 \% 5,6$ los resultados obtenidos previamente con la terapia convencional, no ha tenido el impacto deseado para estas etapas, de ahí los esfuerzos llevados a cabo por diversos autores por incrementar los periodos libres de enfermedad para estos casos y la supervivencia a 5 años sin tumor 1,3,4,7-10. $^{1}$.

Mientras que para el $\mathrm{CaCu}$ avanzado algunos autores recomiendan una terapéutica de consolidación tras la radioterapia y la quimioterapia concomitantes, (RTQTCC) mediante esquemas a base de platino ${ }^{7,11}$ y otros sugieren cirugías complementarias $^{7,9,10}$ o incluso el empleo de quimioterapia previo a cirugías de rescate en pacientes con persistencia o recurrencia tumoral ${ }^{3}$; aún estamos en espera de la terapéutica que permita mejorar ostensiblemente los resultados obtenidos hasta ahora en el tratamiento de esta penosa enfermedad.

En México, las cifras de incidencia y mortalidad del $\mathrm{CaCu}$ entre los cánceres de la mujer solo se ven superadas por las del cáncer mamario y, lamentablemente, más del 60\% de los cánceres invasores se diagnostican en etapas avanzadas, lo que justifica las cerca de 4,000 defunciones que anualmente se registran en nuestro país ${ }^{12,13}$.

Si bien la edad de presentación de las etapas invasoras de la enfermedad es alrededor de los 50 años $^{3,4}$, estudios recientes muestran una tendencia de presentación en edades más tempranas. Se estima que, por lo menos, el $15 \%$ de los casos tiene 40 años de edad o menos ${ }^{14,15}$.

Un informe derivado de la población que acude al Hospital General de México, con 1,217 pacientes $^{15}$, mostró una edad promedio de 51.9 años, con un predominio de los estadios I, con 353 casos (39\%), y el estadio II, con 340 (37\%). El $23.7 \%$ de la totalidad del grupo eran pacientes menstruantes al cursar con 39 años de edad o menos.
En esta publicación se muestran los resultados del tratar miento convencional de RTQTCC en pacientes menstruantes en estadios IB2 y IIA2-IIB de la Federación Internacional de Ginecología y Obstetricia (FIGO) ${ }^{4}$ más cirugías complementarias o de rescate $(C C R)$ en aquellas que mostraron persisten cia tumoral, factores de riesgo para recurrencias tumorales o bien con dichas recurrencias; se llevan a cabo algunas propuestas para el tratamiento integral del $\mathrm{CaCu}$ avanzado, sobre todo en su presentación en mujeres jóvenes, y se ob tienen las conclusiones al respecto.

\section{MATERIAL Y MÉTODOS}

Estudio retrospectivo, observacional y descriptivo de expedientes del archivo clínico del Servicio de Oncología del Hospital General de México Dr. Eduardo Liceaga durante el periodo comprendido de enero del 2010 a diciembre det 2014, seleccionándose para su estudio los de pacientes con $\mathrm{CaCu}$ en estadios clínicos IB2 y IIA2-IIB de la $\mathrm{FIGO}^{4}$ con edades de 45 años o menos, de los que se obtuvo información relacionada con la edad, variedades histológicas (carcinomas epidermoides o adenocarcinomas), terapéutica establecida y resultados de la misma.

La terapia convencional primaria establecida fue de RTQTCC, empleando radioterapia externa a la pelvis a dosis de $50 \mathrm{~Gy}$ en cinco semanas con equipos de teleterapia (acelera dores lineales y con menor frecuencia Cobalto 60), más aplicación de braquiterapia 30 Gy como promedio al término de la teleterapia, para completar un total de 80 Gy. Así mismo, quimioterapia semanal concomitante durante la teleterapia con cisplatino o carboplatino a dosis convencionales, cinco ciclos como promedio ${ }^{1,4}$.

Al término del tratamiento, las pacientes fueron evaluadas por el médico radiooncólogo tratante, quien remitió al Servicio clínico a las pacientes con sospecha de persistencia o progresión tumoral, así como a aquellas con riesgo de recurrencias tumorales por tumores muy voluminosos e histopato logías desfavorables (adenocarcinomas) y a las que por alguna razón no completaron la dosis establecida de braquiterapia ${ }^{1 /}$.

Estas pacientes fueron consideradas para cirugías complementarias a realizarse entre las 6 y 10 semanas posteriores a la terminación del tratamiento, con un promedio de 8 , consistentes en histerectomías radicales clase II sin linfadenectomía pélvica o histerectomías radicales clase III con linfadenectomía a criterio del cirujano ${ }^{3,4,16}$.

Fueron evaluadas para cirugías de rescate consistentes en exenteraciones pélvicas o histerectomías radicales clase 
III ${ }^{4,17}$ las pacientes con persistencia tumoral diagnosticada durante los primeros cinco meses consecutivos a la terminación del tratamiento o con recurrencias tumorales presentadas después de este periodo, previa biopsia positiva para actividad tumoral y con tumor limitado a la pelvis demostrado por tomografía axial computarizada y/o por tomografía por emisión de positrones.

Las pacientes con progresión tumoral no elegibles para cirugías de rescate fueron evaluadas para recibir quimioterapia paliativa con esquemas a base de platinados.

Los resultados obtenidos se sometieron a análisis estadístico con pruebas de $c^{2}$ y prueba exacta de Fisher cuando una de las celdas tenía un valor esperado menor de 5 , con intervalos de confianza del 95\%. Se utilizó el paquete estadístico Info 6.04. Valores de confianza de menos del $95 \%$ fueron considerados sin significación estadística (NS).

\section{RESULTADOS}

\section{Aspectos clinicopatológicos}

Durante el lapso analizado, 400 pacientes con $\mathrm{CaCu}$ avanzado completaron su tratamiento, de las cuales 98 (24.5\%) correspondieron a las etapas IB2, IIA2 y IIB, 23 al estadio IB2 (23.7\%), cinco al estadio IIA2 (5.1\%) y 70 (71.4\%) al estadio IIB. La edad promedio del grupo fue de 37.1 años, el $80.6 \%$ correspondió a carcinomas epidermoides y el $19.3 \%$ a adenocarcinomas (Tabla 1$)$.

\section{Resultados del tratamiento con radioterapia y quimioterapia concomitante}

Setenta y dos pacientes (73.4\%) terminaron su tratamiento sin evidencia clínica ni por imagen de actividad tumoral y 26 (26.5\%) mostraron persistencia tumoral, progresión o recurrencia. Nueve pacientes $(9.1 \%)$ que terminaron su tratamiento sin actividad tumoral abandonaron su seguimiento durante los primeros meses consecutivos a la terminación del tratamiento y no fueron consideradas para los resultados finales.

La evaluación de los resultados finales incluyó a 89 pacientes, de las que 63 recibieron el tratamiento primario (70.7\%) y cursaron de 12 a 62 meses, con un promedio de 30, sin evidencia de actividad tumoral. La cifra incluyó 12 de 19 enfermas en estadio IB2 (63.1\%) y 51 de $70(72.8 \%)$ en estadios IIA-B ( $p=0.3573$ ) (Tabla 2); también 12 de 19 adenocarcinomas $(63.1 \%)$ y 51 de 70 carcinomas epidermoides (72.8\%) ( $p=0.5265$, NS) (Tablas 3 y 4).

El seguimiento sin enfermedad por el lapso mencionado por etapas clínicas de acuerdo a los tipos histológicos fue: para el estadio IB2, cuatro de ocho pacientes con adenocarcinomas $(50.0 \%)$ y ocho de 11 para los carcinomas epidermoides $(72.7 \%)$ ( $p=0.3765 \mathrm{NS})$; para los estadios IIA 2-B, 8 de 11 adenocarcinomas (72.7\%) y 43 de 59 epidermoides (72.8\%) ( $p=0.7819$, NS) (Tablas 3 y 4$)$.

\section{Cirugías complementarias y de rescate}

Dos pacientes con braquiterapia incompleta, más nueve con persistencia tumoral y una con recurrencia fueron consideradas para exploración quirúrgica al evidenciarse clínica-
Tabla 1. Aspectos clinicopatológicos

\begin{tabular}{|c|c|c|}
\hline Variable & Número de pacientes & Porcentaje \\
\hline \multicolumn{3}{|l|}{ Edad en años* } \\
\hline $15-20$ & 6 & 6.1 \\
\hline $21-25$ & 8 & 8.1 \\
\hline $26-30$ & 13 & 13.2 \\
\hline $31-35$ & 11 & 11.2 \\
\hline $36-40$ & 30 & 30.6 \\
\hline $41-45$ & 30 & 30.6 \\
\hline Total & 98 & 99.8 \\
\hline \multicolumn{3}{|l|}{ Tipo histológico } \\
\hline Epidermoides & 79 & 80.6 \\
\hline \multirow[t]{2}{*}{ Adenocarcinomas } & 19 & 19.3 \\
\hline & 98 & 99.9 \\
\hline \multicolumn{3}{|l|}{ Estadio clínico } \\
\hline Estadio IB2 & 23 & 23.4 \\
\hline Estadio IIA2 & 05 & 5.1 \\
\hline Estadio IIB & 70 & 71.4 \\
\hline Total & 98 & 99.9 \\
\hline
\end{tabular}

mente y/o por imagen tumores limitados a la pelvis susceptibles de ser extirpados. Dos casos con persistencia tumoral fueron irresecables (16.6\%), al evidenciar la laparotomía la presencia de metástasis paraaórticas (una paciente en estadio IB2 con adenocarcinoma de células claras y otra con un Ca epidermoide en estadio IIB).

Se llevaron a cabo cinco histerectomías clase II sin linfadenectomía, tres histerectomías radicales clase III y dos exenteraciones pélvicas, una anterior y otra total (Tabla 5). En cuatro pacientes de las 10 resecadas (40.0\%), tres histerectomías clase II y una exenteración pélvica anterior, los informes finales de patología no mostraron residual tumoral. Se incluyen las dos pacientes con braquiterapia incompleta tratadas con histerectomía clase 2, una paciente con histerectomía clase 2 con residual en cérvix y una de las dos pacientes exenteradas. La paciente sometida a una exenteración anterior tenía infor:me previo de recurrencia tumoral de un Ca epidermoide y el espécimen reportó una enfermedad granulomatosa crónica.

Solo en una de las 10 pacientes operadas se obtuvo fracaso terapéutico. Se trató de un adenocarcinoma en estadio IIB con persistencia tumoral en cérvix, tratada mediante una histerectomía radical clase III, cuyo espécimen mostró residual tumoral en cérvix con extensión al istmo uterino sin otros factores pronósticos adversos de riesgo. La paciente desarrolló nueva recurrencia tumoral siete meses después de la cirugía y abandonó su seguimiento.

En los nueve casos restantes se obtuvo un seguimiento entre 13 y 61 meses poscirugía, con un promedio de 30 meses, sin evidencia de enfermedad. La cifra incluyó cuatro de cuatro pacientes en estadio IB2 y cinco de seis en estadio IIB (Tabla 2). Por estirpe histológica, dos de tres, adenocarcinomas y siete de siete, carcinomas epidermoides (Tablas 3 y 4).

Al agregar a los resultados del tratamiento primario RTQTCC los obtenidos con las cirugías ulteriores, observamos 
Tabla 2. Resultados del tratamiento por estadios clínicos

\begin{tabular}{|c|c|c|c|c|c|c|}
\hline \multirow[t]{3}{*}{ Estadio clínico } & \multicolumn{6}{|c|}{ Evolución sin enfermedad* } \\
\hline & \multicolumn{2}{|c|}{$\begin{array}{c}\text { Radioterapia mas } \\
\text { quimioterapia }\end{array}$} & \multicolumn{2}{|c|}{ Cirugía ulterior** } & \multicolumn{2}{|c|}{ Total } \\
\hline & $\begin{array}{l}\text { Número de } \\
\text { pacientes }\end{array}$ & Porcentaje & $\begin{array}{l}\text { Número de } \\
\text { pacientes }\end{array}$ & Porcentaje & $\begin{array}{l}\text { Número de } \\
\text { pacientes }\end{array}$ & Porcentaje \\
\hline Estadio IB2 & (a) $12 / 19$ & 63.1 & $4 / 4$ & 100 & (c) $16 / 19$ & 84.2 \\
\hline Estadio IIA2-IIB & (b) $51 / 70$ & 72.8 & $5 / 6$ & 83.0 & (d) $56 / 70$ & 80.0 \\
\hline Total & (e) $63 / 89$ & 70.7 & $9 / 10$ & 90.0 & (f) $72 / 89$ & 80.8 \\
\hline
\end{tabular}

*30 meses como promedio; ** En pacientes con persistencia o recurrencia tumoral; Significación estadística: (a) vs. (b): $p=0.3573$, (c) vs. (d): $p=0.4714$, (e) vs. (f): $p=0.437$

Tabla 3. Resultados del tratamiento. Adenocarcinomas Adenocarcinomas

\begin{tabular}{|c|c|c|c|c|c|c|}
\hline \multirow[t]{3}{*}{ Estadio clínico } & \multicolumn{6}{|c|}{ Evolución sin enfermedad* } \\
\hline & \multicolumn{2}{|c|}{$\begin{array}{l}\text { Radioterapia mas } \\
\text { quimioterapia }\end{array}$} & \multicolumn{2}{|c|}{ Cirugía ulterior** } & \multicolumn{2}{|c|}{ Total } \\
\hline & $\begin{array}{l}\text { Número de } \\
\text { pacientes }\end{array}$ & Porcentaje & $\begin{array}{l}\text { Número de } \\
\text { pacientes }\end{array}$ & Porcentaje & $\begin{array}{l}\text { Número de } \\
\text { pacientes }\end{array}$ & Porcentaje \\
\hline Estadio IB2 & (a) $4 / 8$ & 50.0 & $1 / 1$ & 100 & (c) $5 / 8$ & 62.5 \\
\hline Estadio Ila-IIB & (b) $8 / 11$ & 72.7 & $1 / 2$ & 50.0 & (d) $9 / 11$ & 81.8 \\
\hline Total & (e) $12 / 19$ & 63.1 & $2 / 3$ & 66.6 .0 & (f) $14 / 19$ & 73.6 \\
\hline
\end{tabular}

Tabla 4. Resultados del tratamiento. Carcinomas epidermoides

\begin{tabular}{|c|c|c|c|c|c|c|}
\hline \multirow[t]{3}{*}{ Estadio clínico } & \multicolumn{6}{|c|}{ Evolución sin enfermedad* } \\
\hline & \multicolumn{2}{|c|}{$\begin{array}{c}\text { Radioterapia mas } \\
\text { Quimioterapia }\end{array}$} & \multicolumn{2}{|c|}{ Cirugía ulterior** } & \multicolumn{2}{|c|}{ Total } \\
\hline & $\begin{array}{l}\text { Número de } \\
\text { pacientes }\end{array}$ & Porcentaje & $\begin{array}{l}\text { Número de } \\
\text { pacientes }\end{array}$ & Porcentaje & $\begin{array}{l}\text { Número de } \\
\text { pacientes }\end{array}$ & Porcentaje \\
\hline Estadio IB2 & (a) $8 / 11$ & 72.7 & $3 / 3$ & 100 & (c) $11 / 11$ & 100 \\
\hline Estadio IIA-IIB & (b) $43 / 59$ & 72.8 & $4 / 4$ & 100 & (d) $47 / 59$ & 79.6 \\
\hline Total & (e) $51 / 70$ & 72.8 & $7 / 7$ & 100 & (f) $58 / 70$ & 82.8 \\
\hline
\end{tabular}

que las cifras globales sin evidencia de enfermedad, que inicialmente fueron del $70.7 \%$, aumentaron hasta el $80.8 \%$, lo que representó un incremento del $10.1 \%(p=0.437$, NS). Para el estadio IB2, las cifras aumentaron desde el $63.1 \%$ al $84.2 \%$ ( $p=0.2690$, NS) y para el estadio II, del $72.8 \%$ al $80.0 \%$ $(p=0.5451$, NS). Para los adenocarcinomas, el aumento fue del $63.1 \%$ al $73.6 \%$ ( $p>0.05$, NS) y para los epidermoides, del $72.8 \%$ al $82.6 \%$ ( $p=0.3255$, NS) (Tablas 2,3 y 4 ).

\section{Fracaso del tratamiento}

En 17 de las 89 pacientes que tuvieron seguimiento (19.1\%) se demostró fracaso de la terapéutica establecida. Estas pacientes formaron parte del grupo de 26 que cursaron con persistencia tumoral, progresión y recurrencia
Tabla 5. Intervenciones quirúrgicas realizadas

\begin{tabular}{|c|c|c|}
\hline Cirugía & $\begin{array}{l}\text { Número de } \\
\text { pacientes }\end{array}$ & Porcentaje \\
\hline Histerectomías clase II & 5 & 41.6 \\
\hline Histerectomías clase III & 3 & 25.0 \\
\hline Exenteraciones pélvicas & 2 & 16.6 \\
\hline Laparotomía y biopsia & 2 & 16.6 \\
\hline Total & 12 & 99.8 \\
\hline
\end{tabular}

(17/26: 65.3\%). La cifra incluye seis de 13 pacientes con persistencia tumoral que presentaron progresión (46.1\%), cinco de ellas con metástasis paraaórticas y la tratada por 
Tabla 6. Fracaso del tratamiento Fracaso del tratamiento

\begin{tabular}{|c|c|c|c|c|c|c|}
\hline \multirow[t]{3}{*}{ Causa } & \multicolumn{6}{|c|}{ Histopatología } \\
\hline & \multicolumn{2}{|c|}{ Adenocarcinomas } & \multicolumn{2}{|l|}{ Epidermoides } & \multicolumn{2}{|l|}{ Total } \\
\hline & Número de pacientes & $\%$ & Número de pacientes & $\%$ & Número de pacientes & $\%$ \\
\hline Persistencia tumoral & $2 / 4$ & 50.0 & $4 / 9$ & 44.4 & $6 / 13$ & 46.1 \\
\hline Recurrencia tumoral & $2 / 3$ & 66.6 & $9 / 9$ & 100 & $11 / 12$ & 91.6 \\
\hline Total $^{*}$ & (a):4/7 & 57.1 & (b): $13 / 18$ & 72.2 & $17 / 25$ & 68.0 \\
\hline
\end{tabular}

Tabla 7. Metástasis a distancia en 13 pacientes

\begin{tabular}{lcc}
\hline Ubicación & $\begin{array}{c}\text { Número de } \\
\text { pacientes* }\end{array}$ & Porcentaje \\
\hline Paraaórticas & 8 & 61.5 \\
Pulmón & 4 & 30.7 \\
Mediastino & 2 & 15.3 \\
Esqueleto & 2 & 15.3 \\
Hígado & 1 & 7.6 \\
Ganglios supraclaviculares & 1 & 7.6 \\
\hline *Cinco pacientes con metástasis en 2 sitios.
\end{tabular}

persistencia tumoral con histerectomía radical que desarrolló recurrencia tumoral y se perdió sin recibir otro tratamiento. Asimismo, 11 de 12 con recurrencias tumorales (91.6\%); cuatro de siete adenocarcinomas (57.1\%) y 13 de 18, carcinomas epidermoides (72.2\%) (Tabla 6). Por etapas clínicas, una de cuatro, estadios IB2 (25.0\%) y cinco de nueve, estadios IIB (55.5\%).

\section{Recurrencias tumorales}

Doce pacientes desarrollaron recurrencias tumorales, que se presentaron entre siete y 28 meses postratamiento primario, con un promedio de 16 meses. Once pacientes fallecieron con actividad tumoral y solo en un caso se obtuvo control sin enfermedad. Se trató de una paciente con un $\mathrm{Ca}$ epidermoide en estadio IIB, que desarrolló recurrencia tumoral 10 meses postratamiento primario y que fue sometida a una exenteración pélvica total. En esta paciente se controlaron 24 meses poscirugía sin enfermedad. Tres pacientes desarrollaron progresión locorregional y fallecieron sin recibir más tratamientos, y ocho diseminación a distancia, cinco de las cuales con actividad locorregional. La ubicación de las metástasis a distancia presentadas por todo el grupo se muestra en la Tabla 7.

Seis de las 17 pacientes en las que fracasó el tratamiento (35.2\%) recibieron quimioterapia paliativa con esquemas a base de platino sin que la terapéutica influyera en su evolución ulterior.

\section{DISCUSIÓN}

El tratamiento de las etapas avanzadas del $\mathrm{CaCu}$, que son las que predominan en países en vías de desarrollo, continúa representando un formidable reto para las instituciones que tienen a su cargo el tratamiento de esta enfermedad ${ }^{1,3,7,12}$.

Si bien el advenimiento de la quimioterapia vino a mejorar en forma global los resultados obtenidos con la radioterapia como terapéutica de elección ${ }^{4,6,18}$, la eventual inac: cesibilidad en países en vías de desarrollo a los modernos equipos de radioterapia y a los fármacos cuya combinación está mostrando mejorar los resultados reportados con el empleo de RTQTCC ${ }^{4,19,20}$ son elementos que contribuyen a que el $\mathrm{CaCu}$ continúe representando una importante causa de muerte para la mujer en países como el nuestro ${ }^{12,13,15,16}$.

La Institución de los autores constituye un Centro de referencia para la población que no tiene acceso a la Seguridad Social, pues solo un $30 \%$ de las pacientes con $\mathrm{CaCu}$ procede de la Ciudad de México; el $60 \%$ de los casos de cánceres invasores se diagnostica en las etapas avanzadas de la enfer-o medad, sin tomar en cuenta los que ya han sido manejados fuera de la Institución. Cuatrocientas diez y ocho pacientes de 908 tratadas bajo el programa de Gastos catastróficos de la Secretaría de Salud del Gobierno Federal (46\%) correspondieron a los estadios IB2 y II de la FIGO ${ }^{15}$, para los que se han reportado entre un 15 y un $23 \%$ de persistencias tumorales o recurrencias con la terapéutica convencional y periodos libres de enfermedad a cinco años, del 75.7 y $65.8 \%$ respectivamente $e^{3,4,20}$. Seleccionamos para su estudio pacientes menstruantes en estadios IB2-IIB tratadas convencionalmente con RTQTCC, por la inquietud derivada de reportes que muestran que por lo menos el $15 \%$ de los casos de $\mathrm{CaCu}$ se presenta en mujeres jóvenes ${ }^{14}$ y el deseo de obtener información respecto a los resultados terapéuticos obtenidos en estas pacientes, considerando que el $23.7 \%$ de las enfermas con $\mathrm{CaCu}$ que acuden a la Institución de los autores tiene 39 años de edad o menos ${ }^{15}$.

Las 98 pacientes de esta serie formaron parte de un grupo de 400 que ameritaron tratamiento convencional RTQTCC y que habiendo sido clasificadas en estadios IB2-IIB tenían edades de 45 años o menos, con un promedio de 37.1 años. Con esta terapéutica se obtuvo un seguimiento sin tumor en 63 de 89 de ellas (70.7\%) durante un periodo de tiempo de 12 a 62 meses, con 30 meses como promedio.

Para el estadio IB2, las cifras fueron de 12/19 (63.1\%); se incluyeron $4 / 8$ adenocarcinomas $(50 \%)$ y $8 / 11$ carcinomas epidermoides (72.7\%). Para el estadio II, 51 de 70 (72.8\%), $8 / 11$ adenocarcinomas $72.7 \%$ y $43 / 59$ (72.8\%), carcinomas epidermoides.

Las cifras referidas para el estadio IB2 son menores al $75.6 \%$ reportado en la bibliografía ${ }^{1,4,6}$, aunque el seguimiento no fue a cinco años. Llama la atención que solo la mitad de las pacientes de este estadio con diagnóstico de adeno- 
carcinoma evolucionó sin evidencia de enfermedad durante el lapso mencionado, situación que habrá de tomarse muy en cuenta para corroborarse o no en un futuro, una vez que se cuente con un mayor número de pacientes con diagnósticos en estadio IB2, aunque los adenocarcinomas representan en nuestra Institución únicamente el $11 \%$ de los casos de $\mathrm{CaCu}^{15}$.

Veintiséis pacientes de la presente serie evolucionaron con persistencia o recurrencia tumorales y 12 de ellas (46.1\%) fueron candidatas a cirugías complementarias, con sospecha o demostración de persistencia tumoral, o a cirugías de rescate por recurrencias tumorales; lográndose elevar mediante estos procedimientos las cifras de seguimiento sin tumor del $63.1 \%$ para los estadios IB2 al $84.2 \%$ (p $=0.2690$, NS) y del $72.8 \%$ al $80.0 \%$ para los estadios II $(\mathrm{p}=$ 0.5451 , NS).

Está bien documentado el valor que tiene la cirugía de rescate para pacientes en las que ha fracasado RTQTCC ${ }^{1,3,4}$, destacando algunos autores la utilidad de llevar a cabo cirugías complementarias no exenterativas en pacientes con sospecha o demostración de persistencia tumoral central ${ }^{7,9,10}$ y otros, la conveniencia de consolidar con mas ciclos de quimioterapia la terapéutica convencional de RTQTCC ${ }^{7,11}$.

En esta serie, 10 de 12 pacientes con sospecha de residual tumoral, persistencia, o recurrencia, fueron sometidas a procedimientos quirúrgicos complementarios o de rescate y solo en un caso se obtuvo fracaso terapéutico, al desarrollar una paciente con persistencia tumoral tratada con histerectomía radical clase 3 una recurrencia tumoral y perderse en estas condiciones. Se realizaron dos exenteraciones pélvicas, con control de la enfermedad. Las cirugías realizadas permitieron mejorar en un $10.1 \%$ los resultados obtenidos previamente con RTQTCC ( $p=0.437, \mathrm{NS})$. Estos resultados recuerdan los referidos en la bibliografía con terapéuticas similares ${ }^{4,7,9,10}$.

Aunque en cuatro casos de los 10 referidos (40\%) los especímenes quirúrgicos mostraron ausencia de residual tumoral, lo cual se interpreta como una respuesta patológica completa a la RTQTCC, preoperatoriamente se tenía documentada la necesidad o conveniencia de llevar a cabo las intervenciones quirúrgicas referidas. En algunas series en las que se ha llevado a cabo un manejo similar se han reportado respuestas patológicas completas hasta de un $60-67.4 \% 9,10$.

Se demostró fracaso de la terapéutica establecida en 17 pacientes de las 89 en que se obtuvo seguimiento (19.1\%). Se incluyen seis de 13 pacientes con persistencia tumoral que presentaron progresión (46.1\%) y 11 de 12 con recurrencias tumorales $(91.6 \%)$ que tuvieron el mismo comportamiento. Cuatro pacientes presentaron actividad tumoral locorregional no controlable y 13 desarrollaron diseminación a distancia. Únicamente seis de las 17 pacientes (35.2\%) recibieron manejo oncológico ulterior sin resultados objetivos. El hecho de que 13 de los 17 fracasos terapéuticos (76.4\%) fueran debidos a diseminación a distancia debe hacernos meditar respecto a la conveniencia de consolidar nuestros tratamientos de RTQTCC con más ciclos de quimioterapia con platino como base, tal como lo están recomendando algunos autores, y de administrar 3-4 ciclos de quimioterapia previo a la exploración quirúrgica de pacientes que serán sometidas a cirugías de rescate ${ }^{3,7,11,20}$.

Un problema que se presenta en la Institución de los autores es la presentación de $\mathrm{CaCu}$ en pacientes jóvenes, pues el $23.7 \%$ de la población que asiste al Servicio con este diagnóstico tiene 39 años de edad o menos ${ }^{15}$ y una buena parte de estas pacientes es tributaria de radioterapia como medida terapéutica de base.

El tratamiento convencional con RTQTCC en pacientes menstruantes suprime el funcionamiento ovárico en forma súbita, con menopausias prematuras, sintomatología vasomotora, pérdida de la densidad mineral ósea, tendencia al sobrepeso y a desarrollar síndromes metabólicos, diabetes mellitus, hipertensión arterial, enfermedad coronaria etc. ${ }^{21,22}$; por lo que resulta justificable para estas enfermas el intentar esquemas de manejo que tiendan a conservar la función ovárica.

Si bien el ascenso de ovarios lejos de los campos de radiación constituye una solución lógica para estas pacientes, habrá que tomar en cuenta otras opciones terapéuticas aplicables en países como el nuestro, donde la demanda de tratamientos de radioterapia suele sobrepasar la oferta de equipos con que cuentan nuestras instituciones ${ }^{16}$. Una de estas opciones la constituye el empleo de quimioterapia neoadyuvante a base de esquemas con platino, seguidas de histerectomía radical, tal y como se recomienda, sobre todo, en países asiáticos ${ }^{14,23-25}$, así como en algunas clínicas de Europa ${ }^{1,6,26,27}$.

La quimioterapia neoadyuvante tiene como finalidad disminuir el volumen tumoral, hacer operables neoplasias que originalmente no lo eran y erradicar o alterar biológicamente las micrometástasis. Debe hacerse notar que las respuestas a los esquemas que utilizan platino como base oscilan entre un 70 y un $100 \%^{1,5,14,23,26,27}$.

\section{CONCLUSIONES}

En nuestra serie la edad promedio de las pacientes fue 37.1 años, el $23.4 \%$ quedó clasificado en estadio IB2 ỹ el $19.3 \%$ del grupo total correspondió a adenocarcinomas.

Sesenta y tres de 89 pacientes con seguimiento tras la RTQTCC (70.7\%) evolucionaron 30 meses como promedio sin evidencia de enfermedad. Se incluye el $63.1 \%$ de los estadios IB2 y el $72.8 \%$ de los estadios II, también el $63.1 \%$ de los adenocarcinomas y el $72.8 \%$ de los carcinomas epidermoides.

Con la terapéutica mencionada la evolución fue menos favorable para los adenocarcinomas en estadio IB2, pues solo cuatro de ocho pacientes (50\%) con este diagnóstico tuvieron seguimiento sin actividad tumoral, frente a ocho de 11 (72.7\%) de los carcinomas epidermoides.

Los resultados fueron similares para ambos tipos histo lógicos en estadio II: ocho de 11 (72.7\%) para los adenocarcinomas y 43 de 59 (72.8\%) para los carcinomas epidermoides.

Con cirugías complementarias o de salvamento, las cifras globales de seguimiento sin evidencia de enfermedad se incrementaron en un $10.1 \%$.

En 17 casos (19.3\%) existió fracaso de la terapéutica establecida. Cuatro por progresión locorregional no controlable $(23.5 \%)$ y $13(76.4 \%)$ por diseminación a distancia.

Si bien la cirugía mejoró en un $10.1 \%$ los resultados obtenidos con RTQTCC, habrá que considerar en un 
futuro el consolidar con esquemas de quimioterapia la terapéutica convencional, con el propósito de disminuir las cifras de recurrencias locales y las de diseminación a distancia.

Es conveniente intentar abordajes terapéuticos diferentes en pacientes menstruantes en estadios IB2-IIB, a fin de evitar menopausias precoces. La quimioterapia neoadyuvante con esquemas a base de platino más histerectomía radical ofrece un campo que vale la pena explorar.

\section{BIBLIOGRAFÍA}

1. Minig L, Patrono MP, Romero N, Rodriguez-Moreno JF, Garcia-Donas J. Different strategies of treatment for uterine cervical carcinoma Stage IB2-IIb. World J ClinOncol. 2014;5(2):86-92.

2. Jemal A, Bray F, Center MM, Ferlay J, Ward E, Forman D. Global Cancer statistics. CA Cancer J Clin. 2011;61(2):69-90.

3. Peiretti M, Zapardiel I, Zanagnolo V, Landoni F, Morrow CP, Maggioni A. Management of recurrent cervical cancer: A review of the literature. Surg Oncol. 2012;21(2):e59-66.

4. Hacker FN, Friedlander LM. Cáncer cervical. En: Berek JS, Hacker NF, editores. Oncología ginecológica $5 .^{a}$ ed. Lippincott Williams\&Wilkins; 2010. pp. 372-76.

5. Sardi EJ, Boixadera AM, Sardi JJ. Neoadjuvant chemotherapy in cervical cancer: a new trend. Cur Open Obstet Gynecol. 2005;17(1):43-7.

6. Buda A, Fossati R, Colombo N, et al. Randomized trial of neoadjuvant chemotherapy comparing paclitaxel, ifosfamide, and cisplatin with ifosfamide and cisplatin followed by radical surgery in patients with locally advanced squamous cell cervical carcinoma: the SNAP01 (Studio Neo-Adjuvante Portio) Italian Collaborative Study. J Clin Oncol. 2005;23(18):4137-45.

7. Shim SH, Lee SW, Park JY, et al. Risk assessment for overall survival in patients with locally advanced cervical cancer treated with definitive concurrent chemoradiotherapy. Gynecol Oncol. 2013;128(1):54-9.

8. Ryu SY, Lee WM, Kim K, et al. Randomized Clinical trial of weekly vs. triweekly cisplatin-based chemotherapy concurrent with radiotherapy in the treatment of locally advanced cervical cancer. Int J Radiat Oncol Biol Phys. 2011;81(4):e577-81.

9. Walji N, Chue AL, Yap C, et al. Is there a role for adjuvant hysterectomy after suboptimal concurrent chemoradiation in cervical carcinoma? Clin Oncol (R Coll Radiol). 2010;22(2):140-6.

10. Colombo PE, Bertrand MM, Gutowski M, et al. Total laparoscopic radical hysterectomy for locally advanced cervical carcinoma (stages IIB, IIA and bulky Stages IB) after concurrent chemoradiation therapy: surgical morbidity and oncological results. Gynecol Oncol. 2009;114(3):404-9.

11. Dueñas GA, Cano BC, Cruz El, Flores TBC. Tratamiento adyuvante y neoadyuvante en cáncer cervicouterino. GAMO. 2014;13(Supl. 4):71-4.
12. Torres-Proveda KJ, Cruz-Valdez A, Madrid-Marina V. Epidemiología del cáncer cervicouterino. GAMO. 2014;13(Supl. 4):4-17.

13. Fernández CSB, Leon AG, Herrera TMC, et al. Perfil epidemiológico de los tumores malignos en México. México D.F.: Secretaría de salud. Subsecretaría de prevención y promoción de la salud. Dirección general de epidemiología. 2011; p 46-122.

14. Hu T, Li Sh, Chen L, Shen J, Li X, Huang K, et al. Matched-case comparison of neoadjuvant chemotherapy in patients with FIGO stage IB-IIB cervical cancer to stablish selection criteria. Eur J Cancer. 2012;48(15):2353-60.

15. Torres LA, Bustamante IJ, Torres RA, Oliva PJC, Morales PMA, Roman BE. Cáncer cervicouterino. Perfil epidemiológico en 1217 pacientes Seguro Popular. GAMO. 2013;81:71-6.

16. Torres LA, Castillo PF, Oliva PJC, Morales PMA, Roman BE. Teleterapia más cirugía en el manejo del cáncer cervicouterino invasor. GAMO. 2009;8:146-51.

17. Torres LA, Lara GC, Oliva PJC, Torres RA, Roman BE, Morales PMA, et al. Laparotomía exploradora por cáncer cervicouterino persistente o recurrente a radiación. Experiencia con 931 pacientes. GAMO 2011;10:335-42.

18. Pettersson F. International Federation of Gynecology and Obstetric (FIGO) Annual Report of the Results of Treatment in Gynecological Cancer. Stockholm: Panorama Press AB; 1998.

19. Tewari KS, Sill MW, Long HJ, et al. Improved Survival with Bevacizumab in advanced cervical cancer. New Engl J Med. 2014;370:734-43.

20. Serrano OJA, Cortés EP, Poitevin ChA, et al. Cáncer cervicouterino: tratamiento de la enfermedad persistente, recurrente o metastásica. GAMO. 2014;13(Supl. 4):75-82.

21. Crandall CJ, Barrett-Connor E. Endogenous Sex Steroid Levels and Cardiovascular Disease in Relation to the Menopause: A Systematic Review. Endocrinol Metab Clin North Am. 2013;42(2):227-53.

22. Murillo-Uribe A, Carranz-Lira S, Martínez-Trejo NA, Santos-González JE: Variables epidemiológicas en la mujer posmenopáusica. Ginecol Obst Méx. 1999;67(10):478-83.

23. Shoji T, Takatori E, Saito T, et al. Neoadjuvant chemotherapy using platinum and taxane based regimens for bulky stage lb2 to Ilb non squamous cell carcinoma of the uterine cervix. Cancer Chemother Pharmacol. 2013;71(3):657-62.

24. Modarress M, Maghami FQ, Golnavaz M, Behtash N, Mousavi A, Khaliti GR. Comparative study of chemoradiation and neoadjuvant chemothe rapy effects before radical hysterectomy in stage Ib-Ilb bulky cervical cancer and with tumor diameter greater than $4 \mathrm{~cm}$. Int Gynecol Cancer. 2005;15(3):483-8.

25. Liu SP, Yang JX, Cao DY, Shen K, Xiang Y, Lang JH. Efficacy of neoadjuvant cisplatin and 5-fluorouracil prior to surgery in FIGO stage Ib2/ IIA2 cervical cancer. Mol Clin Oncol. 2014;2(2):240-4.

26. Gadducci A, Teti G, Barsotti C, et al. Clinicopathological variables predictive of clinical outcome in patients with FIGO stage Ib2-llb cervical cancer treated with cCisplatin-based neoadyuvant chemotherapy followed by hysterectomy. Anticancer Res. 2010;30(1):201-8.

27. Selvaggi L, Loizzi V, Di Gilio AR, Nardelli C, Cantatore C, Cormio G. Neoadjuvant chemotherapy in cervical cancer: a 67 patients experience. Int J Gynecol Cancer. 2006;16(2):631-7. 